\title{
An Evaluation of Models for Predicting Strontium Transport in a Uniform Sand Column
}

\author{
Fumi SUGITA*
}

\section{均一砂層カラム中における Srイオン移動を 予測するモデルの評価}

\author{
杉田 文*
}

\begin{abstract}
For an accurate prediction of contaminant migration in a porous medium, each of the various processes governing solute transport needs to be represented correctly in mathematical models. The transports of many contaminants of environmental concern are influenced by adsorption reaction in the subsurface. In this study, two models commonly used to represent adsorptive solute transport in homogeneous media, namely equilibrium model and two-site kinetic model, were examined for describing strontium transport in a homogeneous sand column. Carefully designed one-dimensional laboratory column experiments were conducted at three different fluid velocities using ${ }^{85} \mathrm{Sr}$ as an adsorptive tracer. Observed breakthrough curves (BTCs) showed asymmetric shape with large dispersion and slight velocity dependence in dispersion and skewness. The equilibrium model which was based on batch $K_{d}$ and a dispersivity of non-reactive tracer showed considerably smaller dispersion and apparently, failed to predict strontium transport in the sand. On the other hand, with a few optimizing parameters, the two-site kinetic model as well as the equilibrium model were able to describe observed BTCs successfully. However, the parameter sets required to describe BTCs for different fluid velocities are inconsistent with theory. In spite of good agreement observed between fitted and observed curves, inconsistent parameter sets indicate that processes occurring in the system are not accurately represented by these models. Since these models are virtually the only ones available to describe inorganic solute transport in homogeneous media, the presence of an additional unknown mechanism which causes considerably large dispersion in reactive BTCs is strongly suggested.
\end{abstract}

\section{Introduction}

It has been a major concern for contaminant

*筑波大学地球科学系, Institute of Geoscience, University, of Tsukuba hydrologists to predict solute migration and first arrival times at certain locations in groundwater systems. Laboratory column experiments are commonly used to investigate solute transport in geologic materials. A number of breakthrough curves 
(BTCs) which describe the profile of the solute concentration of the column effluent have been obtained and used to analyze the mechanisms of solute transport. Transport of non-reactive solute in a homogeneous media is known to be well described by the advection-dispersion equation (ADE). Many contaminants of environmental concern undergo adsorption reaction in subsurface. By assuming local equilibrium condition and singular and linear isotherms, adsorptive solute transport can also be described by ADE with retardation factor $(R)$, using a dispersivity of non-reactive tracer and a batch $K_{d}$ value. However, significant deviation between breakthrough curves (BTCs) observed and predicted by $\mathrm{ADE}$ with $R$ (i.e. equilibrium model) has been reported. Schematic of typical BTCs predicted and observed for non-reactive and reactive solutes is shown in Figure 1. All BTCs in this paper were plotted against dimensionless time called pore volume which is defined as total effluent volume divided by total pore volume in a given column. The observed reactive curves often show more asymmetric shape with larger dispersion than predicted ones, while non-reactive BTCs show good agreement. The curves which deviate from equilibrium model predic-

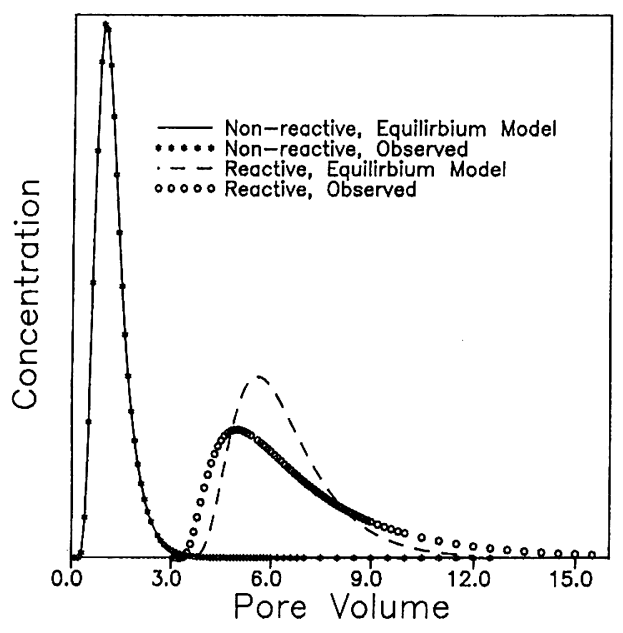

Fig.1. Schematic diagram of typical BTCs observed and predicted by $A D E$ or equilibrium model for non-reactive and reactive sloutes tion are often referred to as 'non-ideal' BTCs. The occurrence of non-ideal BTCs is generally attributed to the violation of one of the underlying assumptions in the adsorption term in the equilibrium model. A comprehensive review of non-ideal BTCs is given by Brusseau and Rao (1989a).

The models which were developed in an attempt to describe non-ideal BTCs can be classified as follows: 1) Physical kinetic model; 2) Intra-organic matter diffusion (IOMD) model; 3) Chemical kinetic model; 4) Non-singular isotherm model and; 5) Nonlinear isotherm model. The first three models describe violation of local equilibrium assumption and their predictions deviate from those of equilibrium model by showing both early breakthrough and longer tailing. Non-singular and non-linear isotherm models describe violation of singular and linear isotherm assumptions respectively and predict either early breakthrough or tailing but not both (van Genuchten, et al., 1974, Brusseau and Rao, 1989a). Most non-ideal BTCs show both early breakthrough and tailing as shown in Figure 1. Although a combination of non-linear and non-singular isotherm models could predict both early breakthrough and tailing, effects of these mechanisms are expected to be insignificant in the range of most groundwater contamination concentrations (Brusseau and Rao, 1989). Therefore non-ideal BTC is often represented by one of the first three models.

Physical kinetic model assumes presence of mobile and immobile regions in the soil and nonideality is attributed to solute diffusion into immobile region. Because this mechanism affects both non-reactive and reactive (adsorptive) solute transports, physical kinetic model predicts non-ideal BTCs not only for reactive solutes but also for nonreactive solutes. IOMD model recently started to receive attention and several researchers demons. trated that it was responsible for the non-ideal behaviour of organic chemicals (Brusseau and Rao, 1989b, Lee et al., 1988, Nkedi-Kizza et al, 1989). Due to different adsorption mechanisms, however, inorganic solutes would not be affected by IOMD (NkediKizza et al., 1989). Chemical kinetic model attributes 
non-ideality to rate limited adsorption reaction in the medium. A commonly used model of this type is two-site model in which one site in the soil is assumed to be in equilibrium while the other site undergoes time dependent adsorption. IOMD and chemical kinetic models predict non-ideal BTCs only for reactive solutes and predict ideal BTCs for non-reactive solutes, because their mechanisms are related to adsorption reaction.

Many non-ideal reactive BTCs accompanied by ideal non-reactive BTCs have been reported, especially for inorganic chemicals in homogeneous media (e.g. Lai and Jurinak, 1972, James and Rubin, 1979, Reynolds et al., 1982). In such cases, chemical kinetics is the only probable mechanism currently available to explain non-ideality. Many of the research on testing applicability of the chemical kinetic model to describe non-ideal BTCs have been focused on organic solute transports. The purpose of this paper is to critically evaluate the chemical kinetic model as well as equilibrium model for prediction of rather simpler case, inorganic solute transport in a homogeneous medium, by applying the models to strontium BTCs obtained in the laboratory.

\section{Theory}

Equilibrium model

One-dimensional adsorptive solute transport in a saturated homogeneous porous medium is described by equilibrium model shown below under the assumptions of local equilibrium, linear and singular isotherms for the reaction:

$$
R \frac{\partial C}{\partial t}=D \frac{\partial^{2} C}{\partial x^{2}}-V \frac{\partial C}{\partial x}
$$

where

$$
\begin{aligned}
& R=1+\frac{\rho}{\theta} K_{d} \\
& D=\alpha_{l} V+D_{0} \tau
\end{aligned}
$$

$C$ is the solute concentration, $t$ is the time, $x$ is the distance, $D$ is the dispersion coefficient, $V$ is the average pore water velocity, $R$ is the retardation fac- tor, $\rho$ is the bulk density of a porous medium, $\theta$ is the porosity, $K_{d}$ is the distribution coefficient, $\alpha_{l}$ is the dispersivity, $D_{0}$ is the molecular diffusion coefficient in free solution and $\tau$ is the tortuosity. Equation 1 represents non-reactive solute transport when $R$ is unity (i.e. $K_{l l}=0$ ). This equilibrium model contains two independent parameters, $R$ and $D$, which are to be obtained prior to simulations. $R$ can be evaluated separately by conducting batch experiments to obtain $K_{d}$ and by measuring bulk density and porosity of the porous medium. The evaluation of $D$ for the non-reactive solute usually involves curve fitting because of the term $\alpha_{l}$. Since the $\alpha_{l}$ value evaluated for non-reactive solute by curve fitting should also be applicable for reactive solute in the same porous medium, $R$ as well as $D$ for reactive solute could be obtained independently.

In a semi-infinite medium, a first type inlet boundary condition yields flux concentration (Parker and van Genuchten, 1984). Thus, boundary and initial conditions for the analytical solution used to describe column effluent in this study were:

$$
\begin{aligned}
& C(0, t)=C_{0}\left(0 \leq t \leq t_{1}\right) \\
& C(0, t)=0\left(t_{1}<t\right)
\end{aligned}
$$

$$
\frac{\partial C}{\partial x}(\infty, t)=0
$$

$$
C(x, 0)=0
$$

where $t_{1}$ is the tracer pulse length.

Two-site chemical kinetic model

When kinetic reaction is approximated to be first-order, two-site chemical kinetic model is expressed as follows (van Genuchten, 1981):

$$
\left(1+\frac{F \rho K_{d}}{\theta}\right) \frac{\partial C}{\partial t}+\frac{\rho}{\theta} \frac{\partial S_{2}}{\partial t}=D \frac{\partial^{2} C}{\partial x^{2}}-V \frac{\partial C}{\partial x}
$$

$$
\frac{\partial S_{2}}{\partial t}=\alpha\left\{(1-F) K_{d} C-S_{2}\right\}
$$

$S_{2}$ is the adsorbed concentration, $F$ is the fraction of reaction sites occupied by equilibrium adsorption 
and $\alpha$ is the first-order rate coefficient. The $D, \rho \theta$ $K_{d}, F$ and $\alpha$ are independent variables in this model. $D, \rho \quad \theta$ and $K_{d}$ can be obtained independently as described previously. On the other hand, $F$ and $\alpha$ are usually evaluated by curve fittings, because they are difficult to measure directly in laboratories with presently available techniques. The same boundary and initial conditions assigned for the equilibrium model was used for this model.

\section{Methods}

The apparatus set up and procedures taken for the column experiments are the same as those described in detail by Reynolds et al. (1982) except that tracers were introduced as pulses rather than continuous sources to reduce amount of waste water containing the tracers. Thus, a brief explanation of the experimental methods is presented below.

Major components of the experimental system are a plexiglass column of $5 \mathrm{~cm}$ long and $6.5 \mathrm{~cm}$ inner diameter with endcaps, a background solution reservoir, a peristaltic pump and an automatic fraction collector. The endcaps of the column were grooved radially to facilitate instantaneous source injection and collection as one-dimensional flow (Reynolds et al., 1982). The column was packed by fairly uniform sand (average particle diameter $0.014 \mathrm{~cm}$ ) and then saturated with simulated groundwater. The material properties and the simulated groundwater composition are listed in Table 1. Non-reactive tracer was labeled by tritium while strontium in the reactive tracer was labeled by trace amount of ${ }^{85} \mathrm{SrCl}_{2}$ (approximately $0.0001 \mathrm{mg} / \mathrm{l}$ ). It is to be noted that the chemical components of the reactive tracer solution are made to be almost identical to that of background solution to provide the same chemical condition for reaction in the two solutions. The distribution coefficient of the strontium in the given sand was reported to be $1.65 \mathrm{ml} / \mathrm{g}$ (Starr et al., 1985) and the calculated R. value based on this batch $K_{d}$ was 10.0 .

All experiments were conducted under steady state flow condition. After steady flow rate was
Table 1. The sand column properties and simulated groundwater composition

\begin{tabular}{lrr|llll}
\hline \multicolumn{2}{l|}{ Sand column properties } & & \multicolumn{3}{l}{ Solution composition (mg/l) } \\
\hline Bulk density & 1.67 & $\mathrm{~g} / \mathrm{cm}^{3}$ & $\mathrm{Ca}^{2+}$ & 39.8 & $\mathrm{Cl}^{-}$ & 62.52 \\
Specific density & 2.62 & $\mathrm{~g} / \mathrm{cm}^{3}$ & $\mathrm{Mg}^{2+}$ & 7.44 & $\mathrm{SO}_{4}^{2-}$ & 2.30 \\
Porosity & 0.36 & & $\mathrm{Na}^{+}$ & 3.10 & $\mathrm{HCO}_{3}^{-}$ & 61.30 \\
One pore volume & 59.98 & $\mathrm{~cm}^{3}$ & $\mathrm{~K}^{+}$ & 2.20 & & \\
& & & $\mathrm{Sr}^{2+}$ & 0.10 & \\
\hline
\end{tabular}

established, the column was flushed with at least twenty pore volume of background solution. Then, the tracer solution was introduced to the column and effluent collection was started at the same time. The collected effluent were analyzed by a beta-particle counter (TRI-CARB, Model 1900CA) for tritium and by a gamma counter (Packard, Auto-Gamma, Model 5650) for strontium-85. A tritium experiment was conducted prior to strontium experiments to ensure absence of apparatus defects and immobile zone in the system. Then, strontium experiments were conducted at three different pore-water velocities $(1.105,0.300$ and $0.076 \mathrm{~cm} / \mathrm{min})$.

\section{Results and Discussion}

The tritium experiment was conducted at average pore-water velocity of $0.300 \mathrm{~cm} / \mathrm{min}$. An obtained BTC and a fitted curve by $\mathrm{ADE}$ are shown in Figure 2. All curve fittings of both $\mathrm{ADE}$ and twosite model analytical solutions (van Genuchten, 1981) to the observed data in this study were performed using a non-linear auto curve fitting computer program, CXTFIT, provided by van Genuchten (1988, personal communication). The observed curve shows fairly symmetric ideal shape, although sampling interval was too sparse, and is in good agreement with the fitted curve of ADE. This indicates that the experimental apparatus and procedure are assumed to provide representative results and that there is no significant immobile zone in the system. Thus, either equilibrium or chemical kinetic model should be able to describe adsorptive solute 


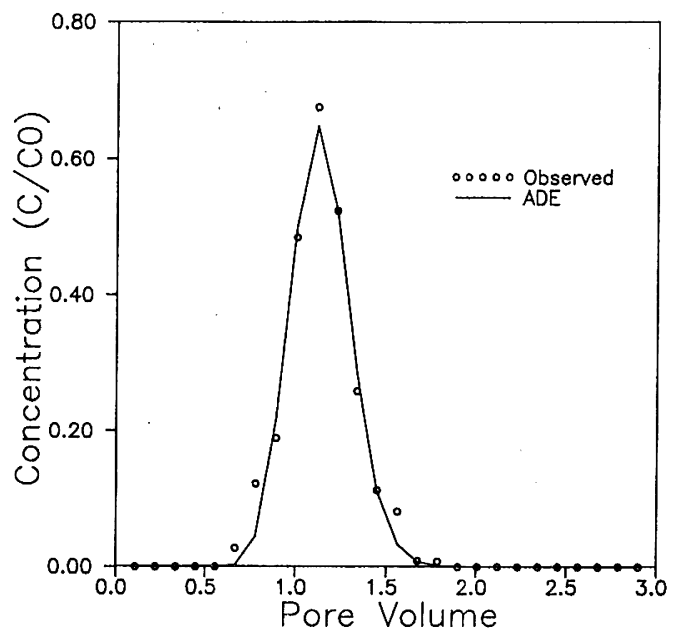

Fig.2. Tritium BTCs observed and fitted by ADE

transport in this system. The optimized dispersivity value of the system was $0.035 \mathrm{~cm}$ which is in a typical range of dispersivity values for uniform sand media in laboratories.

Flow rate in strontium experiments were varied more than one-order of magnitude $(1.105 \mathrm{~cm} / \mathrm{min}$, $0.300 \mathrm{~cm} / \mathrm{min}$ and $0.076 \mathrm{~cm} / \mathrm{min}$ ). Observed BTCs are shown in Figure 3. All three curves show asymmetric shape. Slight velocity dependence in dispersion and apparent skewness are observed, and that both of them increase as velocity increases. The portion of sites governed by equilibrium adsorption in the two-site model decreases as fluid velocity in creases thus, larger skewness and dispersion are expected with velocity increase. The observed properties of strontium BTCs agree with that of two-site model prediction qualitatively.

Equilibrium model predictions based on batch $K_{d}$ and tritium dispersivity values (ideal BTCs) for the three velocities are shown in Figure 4. Predicted curves are symmetric and less dispersed, and it is obvious that equilibrium model failed to describe strontium transport in the sand column. Total mass (area under the curve) of the three pulses are matched to the amount of tracer injected in each experiment. Due to slight difference in the amount of

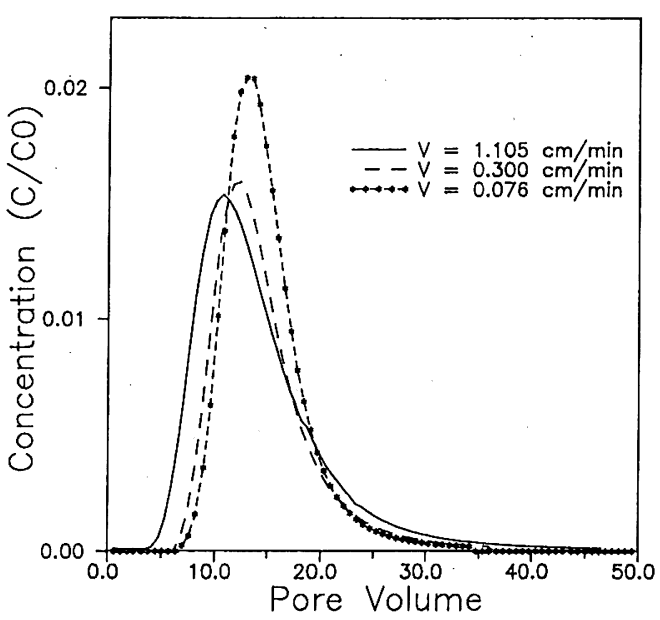

Fig.3. Observed strontium BTCs at three different average pore-water velocities

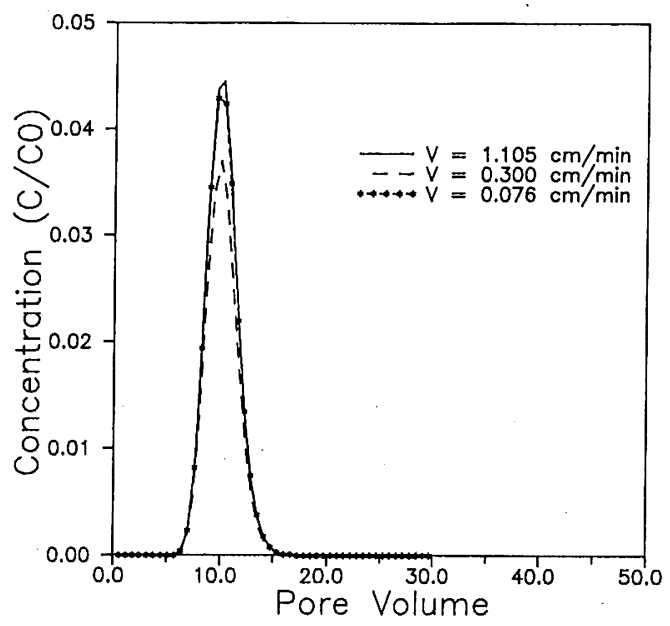

Fig.4. Strontium BTCs predicted by ADE model based on batch $K_{d}$ and tritium dispersivity

tracer solutions, the three curves look different but no velocity dependence in dispersion or skewness is observed.

The analytical solution of two-site model was fitted to observed curves using $K_{d}, F$ and $\alpha$ as fitting parameters, since $F$ and $\alpha$ cannot be obtained independently easily. Equilibrium model predictions indicated batch $K_{d}$ is not valid to describe observed 
curves, thus it was also fitted. The results are shown in Figure 5 (a), (b) and (c). Good matching between observed and fitted curves at all three velocities can be seen. Due to the multiple parameter fitting, however, good agreement does not necessarily indicate that two-site model represents actual process occurring in the system. In order to examine appropriateness of the model, the values of $K_{d}, F$ and $\alpha$ are tabulated in Table 2. Theoretically, $K_{d}$ and $\alpha$ should remain constant regardless of fluid velocity, while $F$ could decrease as $V$ increases. As expected, $F$ increase as $V$ decreases. However $K_{d}$ shifts to larger values and $\alpha$ dramatically changes with decreasing velocity, indicating that these parameter sets are inconsistent with the theory. Thus it could be concluded that two-site model is not adequate to describe strontium transport in the column in spite of good agreement obtained between fitted and observed curves.

Batch $K_{d}$ obtained in a static system and tritium dispersivity values used to evaluate $R$ and $D$ to predict ideal BTCs may not be valid to describe column effluent due to its dynamic nature of the system and possible water chemistry change accompanied by adsorption reaction (e.g. Reardon, 1981). The analy. tical solution of equilibrium model was fitted to the observed data using $R$ and $D$ as fitting parameters, and the results are also shown in Figure 5. With two fitting parameters, the predictions almost perfectly coincide with those of the two-site model and that good agreement with observed curves can be found. Since ADE with batch $K_{d}$ and the tritium $\alpha_{t}$ provided poor fits, it is obvious that this good agreement is obtained due to multiple fitting parameters. Thus, the values of $R$ and $\alpha_{l}$ obtained by optimization need to be examined and they are tabulated in Table 2. Although theoretically, both $R$ and $\alpha_{l}$ values should remain constant regardless of fluid velocity, especially the dispersivity value varies with velocity change in considerable degree. Because of the parameter sets required to fit BTCs for different velocities are inconsistent, equilibrium model itself (without applying batch $K_{d}$ nor tritium $\alpha_{l}$ ) is not adequate to describe strontium transport in a sand
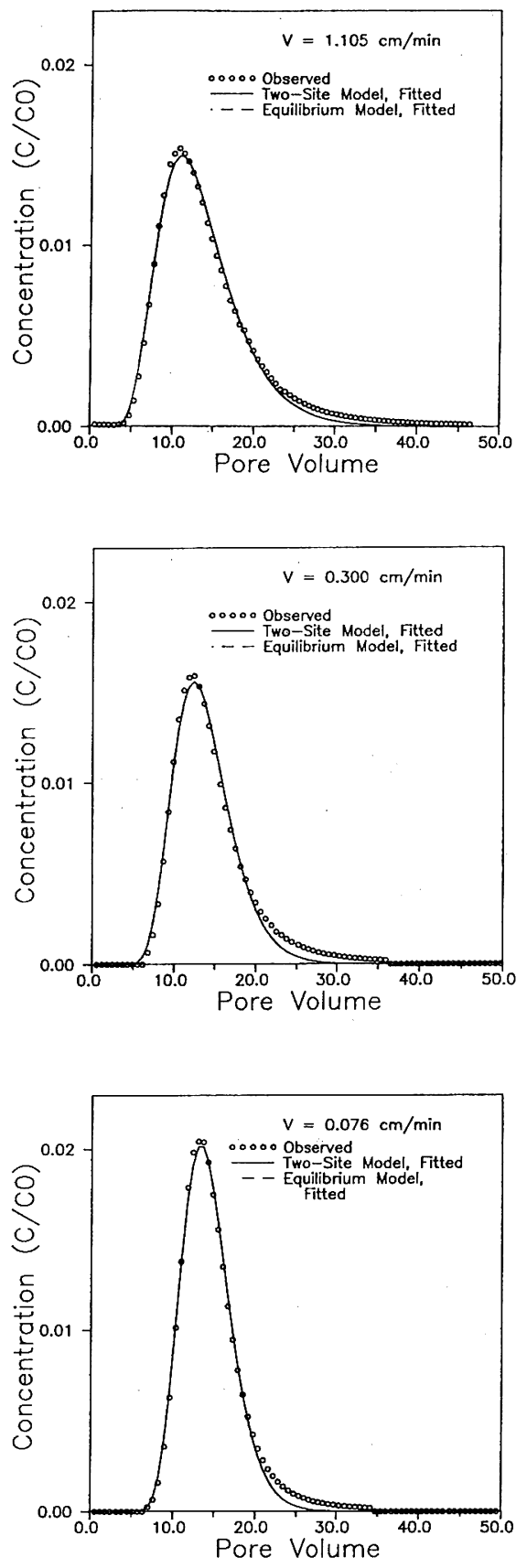

Fig.5. Strontium BTCs observed (open circles), fitted by two-site kinetic model (solid lines) and by equilibrium model (dashed lines). (a) $\mathrm{V}=1.105 \mathrm{~cm} / \mathrm{min}$, (b) $V=0.300 \mathrm{~cm} / \mathrm{min}$ and (c) $\mathrm{V}=0.076 \mathrm{~cm} /$ $\min$ 
Table 2. Parameter values obtained based on best fitted curves for three different velocities for two-site and equilibrium models $\left(K_{d}\right.$ : distribution coefficient, $F$ : portion of sites occupied by equilibrium reaction, $\alpha$ : first-order rate coefficient, $R$ : retardation factor, $\alpha$ : dispersivity)

\begin{tabular}{c|ccc|cc}
\hline Velocity (cm/min) & \multicolumn{2}{|l|}{ Two-Site Model } & \multicolumn{2}{|l}{ Equilibrium Model } \\
\hline & $K_{d}$ & $F$ & $\alpha$ & $R$ & $\alpha_{l}$ \\
& $(\mathrm{ml} / \mathrm{g})$ & $(-)$ & $(/ \mathrm{min})$ & $(-)$ & $(\mathrm{cm})$ \\
\hline 1.105 & 2.27 & 0.486 & 0.0294 & 13.40 & 0.344 \\
0.300 & 2.37 & 0.659 & 0.0101 & 13.65 & 0.169 \\
0.076 & 2.43 & 0.756 & 0.0043 & 14.18 & 0.091 \\
\hline
\end{tabular}

column

Although both two-site and equilibrium models successfully described experimental BTCs, inconsistent parameter sets were found. This indicated that the process actually occurring in the column was not fully explained by these models. Provided that these two are the only models currently available to describe strontium transport in a homogeneous medium, there must be an unaccounted mechanism which causes non-ideal behaviours of reactive solutes in homogeneous media. Since strontium diffusion transport was reported to be well described by the equilibrium model (Gillham et al., 1984), the un known mechanism may be related to advection process. In order to identify the actual mechanisms governing reactive solute transports, properties of reac tive BTCs obtained under various experimental condition should be closely examined.

\section{Summary and Conclusions}

Strontium BTCs were obtained from a uniform sand column at three different fluid velocities. For all three curves, considerably large dispersion with slight velocity dependence in dispersion and skewness was observed. Equilibrium model based on batch $K_{d}$ and a non-reactive tracer dispersivity pre- dicts smaller dispersion and apparently failed to predict observed BTCs. With a few fitting parameters, both equilibrium and two-site models were able to describe experimental curves at different velocities quite well. Inconsistent parameter sets are, however, found to be required to describe the BTCs of different flow velocities in both models. This indicates both models do not represent actual process occurring in the column accurately in spite of their good agreement observed in curve fittings. These are virtually the only available models which predict strontium transport in homogeneous media. Based on these findings, the presence of additional unknown mechanism which causes non-ideal behaviour only for reactive solute transport in homogeneous porous media is suggested. Further sophisticated experimental and modeling studies are required to identify the unknown mechanism.

\section{Acknowledgements}

I' would like to thank Dr. Gillham (Univ. of Waterloo) for his valuable suggestions. I also would like to thank Peggy Evans and Stephanie O'Hannesin (Univ. of Waterloo) for their advises and assistance on conducting laboratory experiments.

\section{Reference}

[1] Brusseau, M.L. and P.S.C. Rao (1989a): Sorption nonideality during organic contaminant transport in porous media. CRC Crit. Rev. Environ. Control, 19, 33-99.

[ 2 ] Brusseau, M.L. and P.S.C. Rao (1989b): The influence of sorbate-organic matter interaction on sorption nonequilibrium. Chemosphere, 18, 9, 1691-1706.

[ 3 ] Gillham, R.W., M.J.L. Robin, DJ.Dytynyshyn and H.M. Johnson (1984): Diffusion of nonreactive and reactive solute through fine-grained barrier materials. Canadian Geotechnical Journal, 21, 3, 541-550.

[ 4 ] James, R.V. and J. Rubin (1979): Applicability of the local equilibrium assumption to transport through soil of solutes affected by ion exchange, in Chemical modeling of Aqueous Systems, edited by Jenne, E.A., 225-235, American Chemical Society, Washington, D.C.

[ 5 ] Lai, S. and JJ. Jurinak (1972): Cation adsorption in one-dimensional flow through soils. Water Resour. Res., 8 , 99-107.

[6 ] Lee, L.S., P.S.C. Rao, M.L. Brusseau and R.A. Ogwada 
(1988): Nonequilibrium sorption of organic contaminants during flow through columns of aquifer materials. Environ. Toxicol Chem., 7, 799-793.

[ 7 ] Nkedi-Kizza, P., M.L. Brusseau, P.S.C. Rao and A.G. Hornsby (1989) : Sorption nonequilibrium during displacement of hydrophobic organic compounds through soil columns with aqueous and mixed solvents. Environ. Sci. Technol., 23, 814-820.

[ 8 ] Parker, J.C. and M. Th. van Genuchten (1984): Fluxaveraged and volume-averaged concentrations in con tinuum approaches to solute transport. Water Resour. Res., 20, 866-872.

[9] Reardon, E.J. (1981): $K_{d}$ 's- Can they be used to describe reversible ion sorption reaction in contaminant migration? Ground Water, 19, 3, 279-286.

[10] Reynolds, W.D., R.W. Gillham and J.A. Cherry (1982):
Evaluation of distribution coefficients for the prediction of strontium and cesium migration in a uniform sand. Canadian Geotech. J., 19, 92-103.

[11] Starr, R.C., R.W. Gillham and E.A. Sudicky (1985): Experimental investigation of solute transport in stratified porous media, 2 . the reactive case. Water Resour. Res, 21, 7, 1043-1050.

[12] van Genuchten, M. Th. (1981): Non-equilibrium transport parameters from miscible displacement experiments, USDA, U.S. Salinity Lab. Res. Rep., 119p.

[13] van Genuchten; M. Th., J.M. Davidson and P.J. Wierenga (1974): An evaluation of kinetic and equilibrium equations for the prediction of pesticide movement through porous media. Soil Sci. Soc. Am. Proc., 38, 29-35.

(received: Oct. 24, 1991, accepted: June 8, 1992) 